\title{
5 Forschungsagenda
}

Um die Ausbreitung von Resistenzen zu verringern und neue Antibiotika $\mathrm{zu}$ entwickeln, sind einerseits stärkere Forschungsanstrengungen und andererseits Rahmenbedingungen erforderlich, die eine effektive Umsetzung wissenschaftlicher Erkenntnisse in die Praxis ermöglichen. Aus Sicht der Akademien sollten Forschungsaktivitäten ein breites Portfolio an Themen und Methoden abdecken, um der Problematik der Antibiotika-Resistenz von verschiedenen Seiten zu begegnen. Forschungsbedarf besteht insbesondere bei Prozessen zur Entwicklung von Assays für die Identifizierung, Validierung und Modifikation von Targets, bei der Entwicklung aussagefähiger Tiermodelle, der Umsetzung von Erkenntnissen aus der Strukturbiologie, bei Analysen zur Struktur-Wirkungsbeziehung und der medizinische Chemie.

\subsection{Genomforschung}

Seit über 15 Jahren ist es möglich, Gesamtgenome von Mikroorganismen $\mathrm{zu}$ entschlüsseln und zu analysieren. Dieser neue methodische Zugang hat die Biomedizin revolutioniert und ist auch bedeutsam für die Forschung im Bereich der Antibiotika-Resistenzen. In den letzten Jahren wurden neue Technologien entwickelt (z. B. das Next Generation Sequencing - NGS), die für die Resistenzforschung zugänglich gemacht werden müssen. Um dies zu ermöglichen, ist der Aufbau einer leistungsfähigen Bioinformatik notwendig. Vor allem kommt es darauf an, große Sequenzpakete zu analysieren und Funktionen zu adressieren. Besondere Bedeutung kommt der Analyse von Veränderungen in den Genomen der pathogenen Mikroorganismen zu, vor allem der Analyse von Punktmutationen. Hierbei hat die Genomforschung einen direkten Zugang zur Virulenz- und Resistenzforschung im Hinblick auf die funktionelle Analyse der Genome.

Sehr wichtig ist auch die Expression von Genen unter In-vivo- und In-vitro-Bedingungen. Hierbei spielt das Deep Sequencing eine Rolle, mit dessen Hilfe auch die Expression kleiner RNA-Moleküle analysiert werden kann. Dieser Zugang sollte unbedingt für die Resistenzforschung und die Analyse der Expression resistenz-relevanter Gene genutzt werden. ${ }^{98}$

Die Genomforschung hat mittlerweile auch die Epidemiologie revolutioniert (genomweite epidemiologische Analyse). Dieser Zugang sollte stärker für die Erforschung der Ausbreitung resistenter Erreger genutzt

98 Wenzel SC \& Müller R (2009). 
werden. Dabei bietet es sich an, auch Methoden der Metagenomanalyse anzuwenden. Im Hinblick auf die Resistenzforschung ist es notwendig, die Expression stoffwechselrelevanter Gene zu verfolgen (Metabolomik), um neue Resistenzmechanismen zu evaluieren. Auf Basis der genannten Methoden muss daran gearbeitet werden, einfache Testsysteme zu entwickeln, um die Analyse von Antibiotika-Resistenzen schneller und sicherer möglich zu machen.

Neue Antibiotika sind aus den Erkenntnissen der Genomforschung noch nicht hervorgegangen. ${ }^{99}$ Das liegt unter anderem an ungünstig aufgebauten Testsystemen und an der Verwendung von Substanzbibliotheken mit zu geringer Vielfalt. ${ }^{100}$ Mit Blick auf die Erforschung neuer Antibiotika sollte deshalb mittels intelligenterer Screening-Systeme das vorhandene Potenzial der Genominformation stärker genutzt werden. Eine systematische und breit angelegte Analyse der vorhandenen potenziellen Targets ist möglicherweise ein Schlüssel für zukünftige Erfolge.

Neue Methoden der Genomforschung, wie etwa das Genome Mining, und Fortschritte bei DNA-Sequenzierungsverfahren erlauben eine immer detailliertere Genomanalyse von Lebensgemeinschaften komplexer ökologischer Nischen und können dazu beitragen, neue potenzielle Wirkstoffe zu identifizieren. So wurden bei der Genomanalyse von Actinomyzeten und Myxobakterien mehr als 25 Gencluster für die Produktion von Sekundärmetaboliten entdeckt.

In diesem Zusammenhang muss auch die Möglichkeit, die Genome von Wirtszellen zu analysieren in den Blick genommen werden. Beispielsweise könnten Stoffwechselfunktionen der Wirtszellen neue Angriffsorte für Wirkstoffe darstellen.

\subsection{Synthetische Biologie}

Dynamisch entwickelt hat sich in den letzten Jahren die Forschungsrichtung der synthetischen Biologie. Sie hat zum Ziel, Lebensprozesse im Labor nachzuvollziehen und (Mikro-)Organismen mit neuen Eigenschaften zu etablieren. Dabei kommt der Möglichkeit Bedeutung zu, große DNA-Bereiche im Labor zu synthetisieren. Neue Methoden, die hier entwickelt werden, müssen der Antibiotika-Forschung zugänglich gemacht werden. Dabei wäre die Synthese großer Stoffwechsel-Determinanten im

99 Payne DJ et al (2007).

100 Payne DJ et al (2007). 
Labor zu verbessern, um so die Antibiotika-Produktion zu optimieren. Beispiele aus der Malariatherapie (Artemisininsäure) stimmen optimistisch hinsichtlich der Anwendung dieser Methoden. Darüber hinaus wird die Synthese großer DNA-Bereiche auch genutzt, um die DNA-Vakzinierung und die DNA-Therapie zu optimieren. Langfristig könnte es möglich sein, im Labor Moleküle mit neuen Eigenschaften zu studieren. Auch hierin liegen große Potenziale für die Entwicklung neuer Antibiotika.

\subsection{Identifikation und Weiterentwicklung von Wirkstoffen und Targets}

\subsubsection{Naturstoffforschung}

Mehr als zwei Drittel aller Antibiotika wurden aus Naturstoffen entwickelt oder sind selbst Naturstoffe. Daher ist zu erwarten, dass in der Natur weitere Wirkstoffkandidaten vorkommen. ${ }^{101}$ Eine wichtige Voraussetzung für die Identifizierung dieser Kandidaten ist der Aufbau neuer Substanzbibliotheken, die eine größere strukturelle Vielfalt aufweisen als viele herkömmliche Bibliotheken. Zum Beispiel konnten interessante Verbindungen in Actinomyzeten aus Meeressedimenten isoliert werden.

Weitere hoffnungsvolle Quellen sind terrestrische und marine symbiontische Lebensgemeinschaften sowie bislang nicht ausreichend erforschte mikrobielle Genera. Neue Forschungsansätze untersuchen bakterielle Symbionten von marinen Schwämmen, Insekten und Pilzen. Aber auch Naturstoffe aus tropischen Pflanzen liefern vielversprechende Kandidaten. ${ }^{102}$ Einige bislang wenig erforschte Gruppen wie die Myxobakterien bilden eine Vielzahl antibiotischer Naturstoffe, wie z. B. Myxopyronine, die ein breites antibakterielles Wirkspektrum besitzen, indem sie durch einen neuartigen Mechanismus spezifisch die RNA-Polymerase von Bakterien hemmen. ${ }^{103}$

\subsubsection{Screeningverfahren}

Die enormen Möglichkeiten der synthetischen Chemie erlaubten immer wieder neue Modifikationen, die ein kontinuierliches Füllen der Anti-

101 Molinski TF et al (2009).

102 Li JWH \& Vederas JC (2009).

103 Mukhopadhyay J et al (2008). 
biotika-„Pipeline“ gewährleisteten. ${ }^{104}$ Die drastische Abnahme der Neuzulassungen von Antibiotika seit Mitte der 1990er Jahre zeigt jedoch, dass die Möglichkeiten, bewährte Strukturen chemisch zu modifizieren, weitestgehend ausgereizt sind. Daher ist die systematische Suche nach Produzenten potenzieller Antibiotika-Leitstrukturen zukünftig der einzig erfolgversprechende Weg, um Antibiotika-resistente Bakterien weiterhin bekämpfen zu können. Auch die Vielfalt diverser ökologischer Lebensräume lohnt eine solche Suche. Um das Potenzial der Naturstoffe zu heben und geeignete Kandidaten in Naturstoffsubstanzbibliotheken zu finden, gilt es, neue und effektivere Screeningmethoden zu entwickeln. Dabei kommt den Methoden der klassischen Mikrobiologie eine besondere Bedeutung zu.

Weitere Wirkstoffe gegen Antibiotika-resistente Bakterien sind möglicherweise unter bereits bekannten Verbindungen zu finden. Die kürzlich eingeführten Antibiotika-Klassen - Oxazolidinone, Lipopeptide und Mutiline - wurden bereits vor mehr als zwei Jahrzehnten entdeckt, aber aufgrund der damaligen Fülle der noch wirksamen Antibiotika nicht weiterentwickelt.

Auch verbesserte Screening-Systeme sowie molekularbiologische Methoden der Produktionsoptimierung von Naturstoffen können dazu beitragen, die Ausbeute bei der Suche nach neuen Wirkstoffen zu verbessern. Zum Beispiel minimiert der Einsatz von Indikatorstämmen, die mehrere Resistenzdeterminanten tragen, die Gefahr der Selektion bekannter Verbindungen und solcher, die durch Kreuzresistenz bereits unwirksam sind. Lohnenswert ist auch das Screening verschiedener Substanzbibliotheken auf antibakterielle Aktivität in Gesamtzell-Assays und nicht in einem targetgerichteten Ansatz.

Das Scheitern vieler targetbasierter Programme ließ Zweifel aufkommen, ob durch derartige Suchprogramme neuartige Verbindungen überhaupt gefunden werden können. Mit der Entwicklung neuer Testsysteme könnte die Erfolgsrate jedoch steigen. Darüber hinaus gibt es AntibiotikaTargets, die bislang nicht ausreichend untersucht wurden und wichtige Stoffwechselwege betreffen, wie die Fettsäuresynthese oder die bakterielle Zellteilung. ${ }^{105}$

104 Eine „Pipeline“ ist die Summe aller Wirkstoff-Kandidaten, welche ein Pharmaunternehmen zu einem bestimmten Zeitpunkt entdeckt oder entwickelt. Definition nach Wikipedia, “Drug pipeline”, http://en.wikipedia.org/wiki/Drug_ pipeline (abgerufen am: 25. Oktober 2012)

105 Fabbretti A et al (2011). 
Chancen bestehen auch bei der Ausschaltung von Stoffwechselfunktionen intrazellulärer Bakterien, die häufig nur noch die genetischen Voraussetzungen für einen eingeschränkten eigenen Stoffwechsel haben und die deshalb stark auf den Stoffwechsel der Wirtszellen angewiesen sind (z. B. Rickettsien, Chlamydien, Listerien). Hier könnte auch der Stoffwechsel der betroffenen Wirtszellen interessante Angriffsorte für antibakterielle Wirkstoffe bieten, die die Vermehrung, möglicherweise auch die Persistenz dieser Bakterien verhindern. Die Erforschung solcher Targets wäre eng an die Bereitschaft gekoppelt, antimikrobielle Wirkstoffe mit schmalem Erregerspektrum zu entwickeln, was vermutlich auch multiple Antibiotika-Resistenzen vermeiden könnte.

Ferner setzen strukturbasierte Modellierungsansätze voraus, dass spezifische Hemmer validierter Targets identifiziert werden. Dabei ist zu berücksichtigen, dass targetbasierte Ansätze im ersten Schritt nicht die Problematik adressieren, Zellmembrane zu überwinden. Auch hierbei haben Naturstoffe erfahrungsgemäß einen Vorteil, da sie evolutionär auf Eigenschaften hin optimiert sind, welche der Anwendung zugutekommen.

Sind neue Leitstrukturen identifiziert, muss der Aspekt der pharmazeutischen Eigenschaften der Wirksubstanzen in der Regel für den Einsatz in der Klinik optimiert werden. Dazu hat die Medizinalchemie in den letzten Jahrzehnten vielfältige Methoden entwickelt, welche die Fragen der Absorption, der Verteilung im Körper, des Metabolismus, der Ausscheidung und eventueller Toxizitäten adressieren (ADME). Diese Aktivitäten müssen unbedingt im Zusammenhang mit der Entwicklung neuer Antiinfektiva gesehen werden, da sie einen entscheidenden Beitrag zur Überführung früher Leitstruktur-Identifizierung in die klinische Anwendung leisten.

Verstärkt sollten Testsysteme entwickelt werden, die Aspekte der Virulenz, der Wirts-Pathogen-Interaktionen und der In-vivo-Essentialität berücksichtigen. Zum Beispiel ist die Verfügbarkeit bestimmter Fettsäuren oder Spurenelemente wie Eisen für das Überleben von Infektionserregern in vivo verschieden von den oftmals standardmäßig eingesetzten In-vitro-Testsystemen. ${ }^{106}$ Metabolische Faktoren wurden bislang zu wenig untersucht, könnten aber attraktive Targets liefern. Allerdings erlauben Erreger-spezifische Stoffwechselwege meist nicht die Entwicklung von Breitspektrum-Antibiotika.

Die zu erwartenden Fortschritte bei der Erreger-spezifischen Diagnostik sollten in Zukunft auch die Entwicklung von Antibiotika mit schmalem Wirkspektrum stärken.

106 Brinster S et al (2009). 


\subsubsection{Chemische Synthese und Strukturbiologie}

Eine weitere wichtige Strategie zur Weiterentwicklung von Antibiotika ist nach wie vor die Verbesserung vorhandener Wirkstoffe. Eine neue Dynamik hat dieser Zugang bekommen, weil Arzneimittel hinsichtlich ihrer Struktur analysiert wurden (u. a. mittels der Röntgenstrukturanalyse) sowie durch die Möglichkeit, Rezeptor-Ligand-Interaktionen zu modellieren. Forschungsbedarf besteht hierbei vor allem im Hinblick auf die Aufklärung von Strukturen. Die Medizinalchemie, die in den letzten Jahren eine Renaissance erfahren hat, stellt hierfür neue Verfahren der chemischen Synthese zur Verfügung. Mithilfe bildgebender Verfahren ist es außerdem möglich, die dynamischen Prozesse der Wirkung von Antibiotika optisch zu verfolgen.

Alle zwischen den 1960er Jahren und dem Jahr 2000 zugelassenen Antibiotika waren, bis auf die Carbapeneme, synthetische Derivate existierender Verbindungen (s. Kapitel 2.5.1). Erst mit Linezolid wurde im Jahr 2000 eine neue Substanzklasse, die Oxazolidinone, vor allem zur Behandlung von MRSA, in die klinische Praxis eingeführt. Bei der sogenannten Derivatisierung bleibt das antibiotisch wirksame Grundgerüst intakt und wird durch verschiedene chemische Gruppen außerhalb dieses Kerngerüstes verändert. Dadurch ergeben sich oft Unterschiede in der Wirksamkeit gegenüber verschiedenen Erregergruppen, in ResistenzEigenschaften sowie im pharmako-chemischen Verhalten.

\subsection{Andere antibiotische Agenzien}

Antimikrobielle Peptide (Defensine) oder Aptamere als wirksame antibiotische Agenzien liefern weitere Ansatzpunkte für die Entwicklung neuer Antibiotika und sollten konsequent weiter erforscht werden. Antibiotische Peptide sind in der Natur eine weit verbreitete Waffe zur Abwehr bakterieller Infektionen. Im August 2011 waren 1.399 antimikrobielle Peptide beschrieben (s. The Antimicrobial Peptide Database). ${ }^{107}$ Viele besitzen eine hohe antimikrobielle Aktivität gegen verschiedene Pathogene. ${ }^{108}$ Eine Substanz, PMX-30063, befindet sich in klinischen Phase-IIStudien. ${ }^{109}$ Aptamere, z. B. gegen Beta-Lactamasen, könnten helfen, die

107 http://aps.unmc.edu/AP/main.php (abgerufen am: 13. April 2012).

108 Hancock RE \& Patrzykat A (2002).

109 Butler MS \& Cooper MA (2011). 
Wirksamkeit von Beta-Lactam-Antibiotika wiederherzustellen. ${ }^{110}$ Bei der Anwendung von Peptiden und Aptameren gilt es, in Zukunft Probleme zu lösen, die beispielsweise die systemische Verteilung, die Stabilität und das Erreichen des Wirkortes betreffen.

\subsection{Molekulare Umweltmikrobiologie}

Bis auf wenige Ausnahmen stellen Antibiotika Naturstoffe dar, die von Mikroorganismen, vor allem von Bakterien und Pilzen, produziert werden. Bei den Produzenten handelt es sich häufig um Isolate aus Umwelthabitaten, vor allem um Bodenmikroorganismen, wie Actinomyzeten oder Bazillen. Neuerdings werden Naturstoffe auch vermehrt aus Schwamm- oder Korallenhabitaten isoliert. Unzureichende Kenntnisse gibt es dabei über die Rolle der Antibiotika in der natürlichen Umwelt. Es ist zu berücksichtigen, dass die Gene für die Synthese von Antibiotika oftmals gekoppelt mit resistenzspezifischen Genclustern vorliegen. Gencluster, die für Produktion, aber auch für Resistenzen kodieren, sind häufig auf mobilen genetischen Elementen, wie Plasmiden, Genominseln oder Transposons lokalisiert. Welche Rolle die Antibiotika selbst auf Gentransferprozesse haben, ist nur ansatzweise verstanden. Fest steht, dass der horizontale Gentransfer zwischen Bodenmikroorganismen und Stämmen, die medizinische Bedeutung haben, eine große Rolle bei der Ausbreitung von Antibiotika-Resistenzgenen spielt. Auch diese Prozesse sind, soweit sie in natürlichen Umwelthabitaten vorkommen, noch weitestgehend unverstanden.

Neben der Analyse einzelner Mikrobenstämme, die die Produktion von und die Resistenz gegen Antibiotika determinieren, stellt das umfassende Studium von Umweltkonsortien, unter anderem mithilfe der Metagenomanalyse, eine weitere Herausforderung für die AntibiotikaForschung dar. So könnten beispielsweise Aussagen zur Bedeutung von Antibiotika in Umwelthabitaten gemacht werden. In diesem Zusammenhang ist auch die Analyse der biologischen Wirkung von subinhibitorischen Antibiotika-Konzentrationen auf den Gentransfer, aber auch auf den Stoffwechsel und das Zusammenspiel von Mikroben in den Habitaten von Bedeutung. Durch die umfassende molekulare Analyse von Umweltkonsortien wäre es so möglich, mehr über die natürliche Bedeutung von Antibiotika zu erfahren, um so die Ausbreitung von Resistenzgenen besser zu verstehen und in der Perspektive auch beeinflussen zu können.

110 Kim SK et al (2009). 


\section{6 Ökologische Aspekte der Antibiotika-Resistenz- entwicklung und der Wirtsflora}

Zukünftig sollten auch die Auswirkungen von Antibiotikagaben auf den Wirt intensiver untersucht werden. Die übermäßige Verwendung von Antibiotika fördert nicht nur Resistenzen, sondern bewirkt zudem Veränderungen der Gesamtheit der körpereigenen mikrobiellen Flora. Langfristig könnte dies auch Erkrankungen wie Diabetes, Fettleibigkeit, Allergien oder chronisch entzündliche Darmerkrankungen fördern. ${ }^{111}$ In diesem Zusammenhang spielt auch der Einsatz von Probiotika eine Rolle. Derartige Mikroben sind in der Lage, als apathogene Stämme Krankheitsprozesse zu beeinflussen und zum physiologischen Gleichgewicht vor allem der Darmflora beizutragen.

Metagenomische Methoden wandten Wissenschaftler in den vergangenen Jahren an, um die natürliche physiologische Flora des Menschen und der Maus hinsichtlich der Zusammensetzung der anzüchtbaren und nicht anzüchtbaren Bakterien zu analysieren. Damit ließ sich die Gesamtheit der Genome (Mikrobiom) und Arten (Mikrobiota) bestimmen. Auch die zeitlichen Schwankungen, die Diätabhängigkeit und der Effekt von Antibiotika auf die mikrobielle Flora wurden erfasst. ${ }^{112}$ Darüber hinaus konnte erstmalig die protektive Mikroflora (Schutz vor Salmonellen oder Clostridium difficile) genauer definiert werden. ${ }^{113}$ Es gelang zudem der Nachweis, dass insbesondere das Kolonmilieu den horizontalen Genaustausch über Plasmide und Phagen zwischen Bakterien besonders begünstigt. ${ }^{114}$

Ausgehend von diesen Erkenntnissen gibt es nun Möglichkeiten, die Gesamtheit der Resistenzgene (das Resistom der Mikrobiota) sowie die Gesamtheit der fakultativ-pathogenen Erreger der Darmflora von Krankenhauspatienten im Voraus zu identifizieren. Insbesondere sollten Vancomycin-resistente Enterokokken und extra-intestinal-pathogene E. coli (Pathotyp ExPEC) erfasst werden. Ähnlich wie die Differenzierung von E. coli in mehr als zehn verschiedenen Pathotypen erfolgreich erarbeitet wurde, besteht jetzt die Möglichkeit, auch die übrigen im Darm vorkommenden Arten der Familie der Enterobakterien nach Pathotypen zu differenzieren und ihr nosokomiales Pathogenitätspotenzial vorherzusagen.

111 Blaser M (2011).

112 Costello EK et al (2009); Buffie CG et al (2012); Jernberg C et al (2007); Jakobsson HE et al (2010); Blaser M (2011).

113 Blaser M (2011); Stecher B \& Hardt WD (2011).

114 Stecher B (2012). 
Für die Infektionsmedizin ließe sich so das Risiko von Krankenhauspatienten für endogene Infektionen einschließlich Antibiotika-Resistenzen vorherbestimmen. ${ }^{115}$

Die Mechanismen des horizontalen Gentransfers wie Transposition, Transformation, Transduktion und Konjugation sind schon seit vielen Jahren bekannt. Wichtige daraus resultierende Fragen knüpfen sich an die Mechanismen des Selektionsdrucks, die Induktoren der Genausbreitung und Persistenz sowie an die Möglichkeiten, diese Mechanismen zu kontrollieren oder zu unterbinden.

Inzwischen wurde experimentell belegt, dass subinhibitorische Antibiotika-Konzentrationen, wie sie im Abwasser von Industrieanlagen und Klärwerken auftreten, zur Selektion Antibiotika-resistenter Bakterien beitragen. ${ }^{116}$ Darüber hinaus können subinhibitorische Konzentrationen von Antibiotika über die Bildung von Sauerstoffradikalen die Mutationsrate erhöhen und damit z. B. das Substratspektrum von Beta-Lactamase erweitern und sogar den horizontalen Transfer von Resistenzgenen auslösen. ${ }^{117}$ Neuesten Erkenntnissen zufolge ist konjugativer Plasmidaustausch im entzündlichen Darm (z. B. bei Salmonellose) besonders effizient. ${ }^{118}$ Diese Beobachtungen geben Anlass dazu, traditionelle Therapieempfehlungen für Antibiotika zu überprüfen, die als Monotherapie und häufig in Unterdosierung etabliert sind.

Deshalb fordern Mikrobiologen neben Antibiotika zur direkten Eliminierung der Erreger auch Mittel zur Kontrolle der Ausbreitung von Antibiotika-Resistenzen (Penetranz, Promiskuität, Persistenz und Plastizität). ${ }^{119}$ Mittel gegen diese vier „P“ werden als Eco-Evo Drugs bezeichnet. Sie sollen die Modifikation, Neuentstehung und Ausbreitung von Antibiotika-Resistenz in der Umwelt eingrenzen. $\mathrm{Zu}$ diesem Zweck wurden bereits Plasmidkonjugationshemmer, Bakteriozine und Phagen in vitro eingesetzt. ${ }^{120}$ Dieser Weg klingt vielversprechend und könnte bei Patienten die Mikrobiota des Gastrointestinaltraktes - der Hauptumschlagplatz von Antibiotika-Resistenz - unter Kontrolle bringen.

115 Littmann DR \& Pamer EG (2011).

116 Aminov RI (2009); Davies J et al (2006); Gullberg E et al (2011).

117 Kohanski MA et al (2010).

118 Stecher B et al (2012).

119 Baquero $\mathrm{F}$ et al (2011).

120 Baquero F et al (2011); Lujan SA et al (2007). 


\subsection{In-vitro-Empfindlichkeit versus In-vivo-Wirksamkeit}

Die Antibiotika-Resistenz-Testung bakterieller Patientenisolate erfolgt nach standardisierten In-vitro-Methoden (Nähragar oder -lösung) unter aeroben Bedingungen und exponentiellem Wachstum. Diese konventionellen Testmethoden entsprechen nur selten den Wachstumsbedingungen der Erreger in vivo. Hierbei handelt es sich nicht nur um ein pharmakokinetisches Problem, sondern vielmehr darum, die unterschiedlichen Wachstumsbedingungen und Zustände der bakteriellen Infektionserreger im Patienten zu berücksichtigen. ${ }^{121}$

Bakterien können sich intrazellulär oder extrazellulär vermehren, sie können planktonisch/dispers oder biofilmartig in Mikrokolonien im Gewebe wachsen. Die Sauerstoffspannung am Infektionsort (aerob, microaerophil, anaerob) sowie das Nährstoffangebot bestimmen den Stoffwechsel (Metabolismus) und den Aufbau der äußeren Hülle der Bakterien und damit auch die Empfänglichkeit für Antibiotika. Diese Parameter werden bei der konventionellen Antibiotika-Resistenz-Testung nicht berücksichtigt. Dies beeinträchtigt den prädiktiven Wert der In-vitro-Antibiogramme, wodurch sich das Versagen von Therapien erklären lässt. ${ }^{122}$

Um die Antibiotika-Therapie von Pseudomonas-Infektionen bei $\mathrm{Mu}$ koviszidose/Zystischer Fibrose (CF) zu verbessern, haben Wissenschaftler bereits überzeugende Vorarbeiten geleistet: Sie passten AntibiotikaResistenz-Testmedien an den CF-Bronchialschleim an, schufen anaerobe Bedingungen und berücksichtigten die Biofilmbildung. ${ }^{123}$ Auch für endogene Infektionen mit $E$. coli (vom intestinalen Kommensalen zum Erreger von Harnwegsinfektion bzw. Sepsis) liegen erste Daten über einen veränderten Metabolismus und Lebenszyklus im extra-intestinalen Milieu vor. ${ }^{124}$

Chronische und wiederkehrende (rezidivierende) Infektionen treten nicht selten trotz Antibiotika-Therapie auf. Hierfür werden Persister-Bakterien verantwortlich gemacht, die einen stark reduzierten Metabolismus haben und sich deshalb der Antibiotika-Therapie entziehen können. Erste metabolische Untersuchungen haben gezeigt, dass durch die Fütterung von Escherichia-coli-Persistern mit Glucose oder Pyruvat die Wirkung von Aminoglykosid stark ansteigt. ${ }^{125}$

121 Brown SA et al (2008).

122 Brook I \& Gilmore JD (1993).

123 Sriramulu DD et al (2005); Hill D et al (2005); Dubreuil L \& Odou MF (2010).

124 Alteri CJ \& Mobley HL (2012).

125 Allison KR et al (2011). 
Diese neuen Ansätze, die den Metabolismus und die Lebensweise des Erregers unter Infektionsbedingungen (z. B. Mausinfektionsmodell) berücksichtigen, sind vielversprechend hinsichtlich neuer Therapiestrategien und Antibiotika-Resistenz-Testverfahren. ${ }^{126}$

Darüber hinaus ist es notwendig, klinische Analysen von Therapieversagen, z. B. auf Intensivstationen oder bei der Behandlung von Implantatinfektionen zu verbessern, etwa durch interdisziplinäre Kooperationen zwischen Infektiologen, Mikrobiologen und Pharmazeuten.

Aufgrund der positiven Erfahrungen mit der Verzögerung zukünftiger Resistenzbildungen nach der Einführung neuer antibiotischer Substanzen in die Tuberkulose- und HIV-Therapie besteht großer Forschungsbedarf zum vorrangigen Einsatz neuer Antibiotika als Kombinationstherapeutika zu bestehenden Therapieregimen. Die Auswirkungen dieser Strategie auf die Resistenzbildung auch bei Kurzzeitbehandlungen von Infektionen sind bisher weitgehend unerforscht.

\subsection{Nebenwirkungen von Antibiotika: Wirkung auf das Immunsystem und auf die Erregervirulenz}

Pharmakotoxische Nebenwirkungen von Antibiotika, wie auf dem Beipackzettel aufgeführt (z. B. Nephro- und Ototoxizität von Aminoglykosiden), werden in der Regel beachtet. Weniger bekannt sind die entzündungshemmenden oder immunsuppressiven Wirkungen von Antibiotika, die eine erfolgreiche Infektionstherapie vortäuschen können.

So wirkt Doxycyclin zum Beispiel als Hemmer von Metalloproteasen, als scavenger (d.h. als Auskehrer) für Sauerstoffradikale und als Neuroprotektivum, indem es den programmierten Zelltod hemmt. ${ }^{127}$ Ähnliche antiinflammatorische Wirkungen haben Makrolid-Antibiotika. ${ }^{128}$ Dagegen sind die immunmodulatorischen Wirkungen von Fluorchinolonen komplexer und noch wenig verstanden. Sie können die Blutbildung (Hämatopoese) steigern, eine Form des programmierten Zelltods (Apoptose) und die Ausschüttung des Signalstoffs TNF (Tumornekrosefaktor) hemmen sowie die Synthese des Botenstoffs Interleukin-2 bewirken. ${ }^{129}$

Die Wirkung von Antibiotika auf Bakterien kann bakterizid oder bakteriostatisch sein. Bakterizid wirkende Antibiotika führen zur Bak-

126 Lee HH \& Collins JJ (2012).

127 Griffin MO et al (2011).

128 Amsden GW (2005).

129 Dalhoff A \& Shalit I (2003). 
teriolyse und zur Freisetzung von Substanzen, sogenannte microbial/ pathogen associated molecular pattern (MAMP/PAMP), die z. B. über Tolllike-Rezeptoren (TLRs) proinflammatorische oder antiinflammatorisch wirkende Zytokine und Chemokine auslösen, die die Symptomatik der Infektion und das Ausmaß der Organschädigung bestimmen. ${ }^{130}$ Bisher beschränkten sich die Untersuchungen auf Zellkulturmodelle und artifizielle Maus-Infektionsmodelle. Nur in wenigen Fällen wurden Vergleiche mit definierten Knockout-Mäusen (z. B. TLR $4^{--}$, Myd88 ${ }^{-/}$) durchgeführt. ${ }^{131}$ Da mittlerweile Knockout-Mausmodelle für alle bekannten PAMP-Rezeptoren und Signaltransduktionskomponenten zur Verfügung stehen, sollten die Auswirkungen einer Antibiotika-Therapie auf die angeborene Immunantwort der Infektionen in der Maus mit angepassten Modellerregern gezielt untersucht werden. Solche Untersuchungen könnten auch die Basis für eine personalisierte Therapie von Infektionskrankheiten sein, z. B. wenn Defekte im TLR-System bekannt sind.

Ein weiterer Aspekt der Erforschung der Antibiotika-Wirkung befasst sich mit der regulativen Wirkung subinhibitorischer Konzentrationen auf die Virulenzexpression. Das klassische Beispiel dafür ist die Shigatoxin(ST)-Induktion bei enterohämorrhagisch wirkenden Escherichia coli (STEC, EHEC, HUSEC) durch die Gabe von Antibiotika. ${ }^{132} \mathrm{Be}$ sonders subinhibitorische bakterizid wirkende Antibiotika erzeugen eine Stressantwort (inklusive SOS-Antwort), die $\mathrm{zu}$ einer Aktivierung von Prophagen-Genen (inklusive stx-Gen) führt. Darüber hinaus deuten Micro-Array-Transkriptom-Analysen bakterieller Erreger nach subinhibitorischer Antibiotika-Behandlung auf eine komplexe Genregulation hin, die die Pathogenität und Entwicklung der Antibiotika-Resistenz beeinflusst. ${ }^{133}$

\subsection{Hemmung von Virulenzfaktoren}

Eine alternative Strategie zum herkömmlichen Ansatz, Bakterien durch Antibiotika abzutöten, zielt auf die Hemmung (Inhibition) von Virulenzfaktoren. Diese Strategie lohnt, intensiver erforscht $\mathrm{zu}$ werden, da die spezifische Hemmung von Toxinen oder Adhäsinen eines Erregers eine Infektion möglicherweise verhindert oder abschwächt, ohne nützliche

130 Ginsburg I (2002); Vianna RC et al (2004).

131 Trautmann M et al (2010); Weighardt H \& Holzmann B (2007).

132 McGannon CM et al (2010); Mellmann A et al (2011).

133 Davies J et al (2006); Fajardo A \& Martínez JL (2008). 
Bakterien zu schädigen. Diese Strategie unterstützt natürliche Abwehrmechanismen und würde weniger Nebenwirkungen verursachen. ${ }^{134}$

Vor allem bei Erregern, bei denen ein einzelner Virulenzfaktor für die Auslösung der Krankheit essenziell ist, könnte ein derartiger Ansatz erfolgreich sein. Neben der Disposition der Patienten ist bei vielen nosokomialen Erregern eine Reihe von Virulenzfaktoren am Infektionsgeschehen beteiligt, sodass das Ausschalten eines einzelnen Faktors kaum erfolgversprechend sein dürfte. Voraussetzung für einen solchen Therapieansatz ist jedoch eine schnelle und zuverlässige Diagnostik, die nicht nur den Erreger zweifelsfrei identifiziert, sondern auch die entsprechenden Virulenzfaktoren. Moderne chipbasierte Diagnostiksysteme könnten dies gewährleisen, sind allerdings gegenwärtig noch nicht verfügbar.

\subsection{Sozio-ökonomische Forschung}

Die sozio-ökonomischen, rechtlichen und ethischen Rahmenbedingungen für die Entwicklung neuer Antibiotika sollten stärker erforscht, Hemmnisse identifiziert und Lösungswege aufgezeigt werden. Maßnahmen sollten verstärkt vorausschauend und rückblickend evaluiert werden. Ferner kann sozio-ökonomische Forschung dazu beitragen, Maßnahmen zur schnelleren und effizienten Entwicklung und Anwendung von Antibiotika zu entwickeln. Weiterhin sollten Schwerpunkte auf das Design adäquater Anreizmechanismen und die Untersuchung ihrer Wirkungen gelegt werden, sowie auf ein besseres Verständnis des Entscheidungsverhaltens von Anwendern und Produzenten. Eine große Bedeutung für die schnelle Umsetzung wissenschaftlicher Erkenntnisse in die klinische Praxis kommt den klinischen Studien und der Versorgungsforschung zu.

134 Cegelski L et al (2008). 
